\title{
A new journal
}

\author{
Bill Noble
}

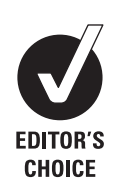

BMJ Supportive \& Palliative Care is a journal for clinicians, researchers and other healthcare workers in all clinical services where supportive and palliative care is practised. The journal aims to link many disciplines and specialties throughout the world; promoting an exchange of research evidence and innovative practice by presenting high quality scientific reports, reviews, comment, information and news of international importance.

The contagion of medical journalism has become endemic in 171 years since Dr Peter Hennis Green and Dr Robert Streeten introduced the BMJ's forerunner, the Provincial Medical and Surgical Journal. ${ }^{1}$ In 1840 , few could have imagined the volume or variety of medical publishing on offer today. It is customary, in the first editorial of any new journal, to answer the question "Why another journal?'

Some doctors spend their working lives and intellectual energy attempting to ease the distress of patients and their carers. In 2011, in a variety of cultures and institutions, along with other kinds of professional, their work may be categorised as palliative care, supportive care, end-of-life care or terminal care. Many other doctors practise with these objectives within a medical specialty that remains focused on curative or life-prolonging measures. Doctors in primary care or family medicine often see this work as an important element of their role, and it offers some of the most challenging and rewarding clinical problems, particularly in the context of other competing priorities.

The ability of healthcare professionals to ease the burden of severe disease of one sort or another depends not only on skills and technology aimed at cure or extending longevity. We also require knowledge of the impact and consequences of disease, and in addition, we need to understand the mechanisms by which our diagnosis and the patient's suffering are linked. Skills that enable doctors to elicit the particular nature and extent of distress in an individual are not

Correspondence to Bill Noble, Academic Unit of Supportive Care, University of Sheffield, Sykes House, Little Common Lane, Sheffield, UK; bill.noble@ sheffield.ac.uk necessarily the same as those required to elicit a diagnosis or give information about treatment. We require access to the latest detailed information on the range of therapeutic measures with potential to intervene effectively.

Understanding the diversity of patients and carers' experience as well as the diversity of response to treatments allows us to plan care and communicate what to expect as disease progresses. At an organisational level, national policy and healthcare institutions that aim to support and care for patients with life-limiting disease require different characteristics from those that only offer services devoted to fixing conditions in discrete episodes of care. In these subtle, but important respects, the concepts of supportive and palliative care bring new emphases in medical practice with new challenges for education and new areas of uncertainty for research.

The first journal devoted to our subject, was Palliative Medicine, published in 1987. Even then its editor, Derek Doyle, felt the need to mention the proliferation of terms describing services that we would now label as a supportive or palliative care. ${ }^{2}$ Medicine has always tended to rename or reclassify problems with no clear solution, so perhaps it is inevitable that every decade brings changes in the words that denote medical activity aimed at the relief of distress in the face of life-threatening illness. Although last year, terminal care was on the wane, papers on supportive care, palliative care, hospice and end-of-life care continued to be published in increasing numbers. ${ }^{3}$ So it appears that there is room for a new title, even though there are several international journals in the field. Some are primarily aimed at the academic readership, some specialise in particular strands of practice, but most accept reports concerning research from

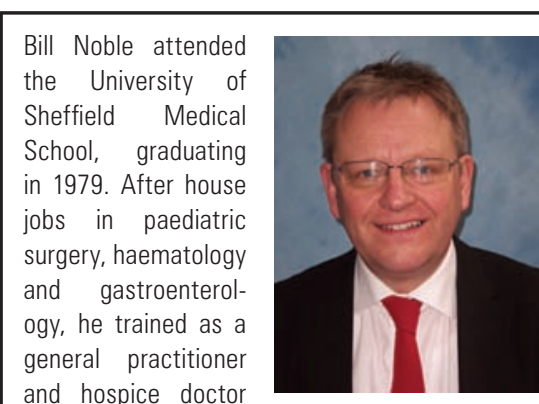
and hospice doctor

before becoming a principle in general practice in a deprived area of Sheffield. He also took up a post as associate specialist in palliative medicine at St Luke's Hospice. During this time, he began academic work at the Trent Palliative Care Centre, teaching communication skills and studying for his $\mathrm{MD}$ on a trial in a home palliative care service.

Bill's current post is Macmillan Senior Lecturer in Palliative Medicine at the University of Sheffield. His clinical work as a consultant physician in palliative medicine informs his research and teaching. Research interests include communication skills training, service evaluation, medical humanities, testing holistic needs assessments and developing palliative care in the community.

He has worked on various committees and groups promoting and governing academic palliative care for organisations such as Macmillan Cancer Support, Marie Curie Cancer Care, the National Cancer Research Institute, the Royal College of Physicians and Dimbleby Cancer Care. As chairman of the Association for Palliative Medicine of Great Britain and Ireland from 2007 to 2010, Bill co-ordinated a strategic review, to take account of developments in the field of palliative care and the changing role of the specialty.

Although he realises that it appears less than adventurous, Bill still lives in Sheffield with his wife and two teenage children. He is prepared to travel, particularly if it involves jazz, fly-fishing, the visual arts or certain aspects of gastronomy.

a wide variety of disciplines. There are review journals as well as those disseminating opinion and accounts of experience in clinical services. Our field is particularly rich in monographs and the grey literature where there is no shortage of new ideas as well as opinions on old ones.

Twenty-four years ago, UK physicians practising palliative medicine had just about agreed that the specialty was here to stay, although UK institutional and health service policy was slower to come to that conclusion. Since then it has been important to define a role and a particular contribution to medicine, in doing so, it was customary to emphasise the specialness of the specialty. Now may be the time to explore what we share with colleagues and look beyond the boundaries of hospice, specialised clinical teams and dedicated research units. Diversity of healthcare systems and cultural attitudes 
to illness and death may produce considerable variation in the way supportive and palliative care needs are addressed around the world, but the opportunities to share insights and disseminate practice might be enhanced by recognition of some of the challenges and questions that are common to practitioners in this field.

There remain areas of study that are in serious need of progress. In particular, clinical research on the control of symptoms is required to replace those parts of treatment protocols still populated by expert opinion. Here, the scope for head-to-head clinical trials of current therapeutic regimes is immense. Systems for introducing patient centred care and facilitating community care are stunted for the lack of evidence of their effectiveness. There remains an important minority of patients in severe pain for whom current therapy does not work and new pharmacological approaches are needed. Certain symptoms such as delirium, cachexia and fatigue remain very difficult to palliate in practice. Even the nature of our uncertainty when faced with the challenge to prognosticate in advanced disease appears unclear. Research and training on skills for communicating with patients has progressed at a better pace than many areas of study, but the impact on clinical outcomes remains unknown and professional communication with family and carers lags far behind.

Resources for supportive and palliative care research have never approached those devoted to studies with curative aims. The commercial return on investment in research and development of therapies used at the end of life will always be limited. So the potential for new knowledge arising from the basic sciences to translate into clinical tools for palliative care may remain obscure for the lack of a driving impetus from the pharmaceutical industry. ${ }^{4}$ With the global financial downturn, some governments are funding less science and charitable grant giving bodies are shrinking into their core business. There is more pressure than ever to make every study count and the quality of research together with its reporting needs to improve quickly.

The BMJ brand stands for a remarkable combination of a lively journalistic style and rigorous peer review. It offers a unique opportunity to combine many elements that might attract the attention of a wide variety of practitioners, researchers and service developers. We aim to reach out and tap the expertise of colleagues wherever new insights, therapies or organisations are studied. I hope BMJ Supportive \& Palliative Care will stimulate collaboration between healthcare professionals in different specialties as well as researchers with different backgrounds. We intend to welcome contributions from many different academic and clinical disciplines while upholding the standards of good science and good writing

\section{REFERENCES}

1. Green PT, Streeten R. Introductory address. Prov Med Surg J 1840;1:1-4.

2. Editorial. Palliat Med 1987:1.

3. Bennett MI, Davies EA, Higginson IJ. Delivering Research in end-of-life care: problems, pitfalls and future priorities. Palliat Med 2010;24:456-61.

4 Winslow M, Paz S, Clark D, et al. Pharmacogenetics and the relief of cancer pain. Int J Technol Knowledge Sci 2007;2:129-34

\section{Competing interests None.}

Provenance and peer review Not commissioned; internally peer reviewed.

Published Online First 13 April 2011

BMJ Supportive \& Palliative Care 2011;1:1-2 doi:10.1136/bmjspcare-2011-000033

\section{A word from two of our Associate Editors}

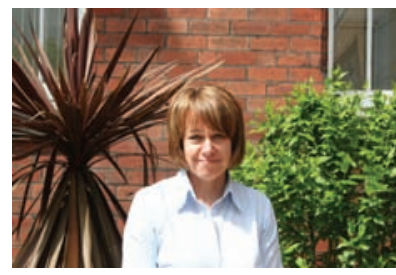

Christine Ingleton

Professor in Palliative Care Nursing School of Nursing and Midwifery The University of Sheffield
As a nurse I am looking forward to joining the journal as an Associate Editor working with an international team of academics, clinicians and policy makers from a variety of different disciplinary perspectives. One of our major challenges for the future is to find ways to improve equity of access to good quality care during the end of life period. Advances in medicine and health technologies means that greater numbers of people will survive for longer with complex health and social care needs. The science of palliative medicine and palliative care nursing is going to be challenged by the management of highly complex symptomology of multiple diseases. This means we need to explore new and innovative ways of greater partnership working and finding solutions to breaking down existing specialisms. Greater prominence needs to be given to other team members such as therapists, spiritual experts, nutritionists, to name but a few, as their skills become better recognised and the evidence base for their efficacy more established. Finally it is hoped that we can engage with issues facing palliative care in resource-poor countries. While lessons can undoubtedly be learnt and adapted from palliative developments in resource-rich countries, lessons can also be learnt from the challenges facing resource-poor countries where there are shining examples showing palliative care at its creative best.

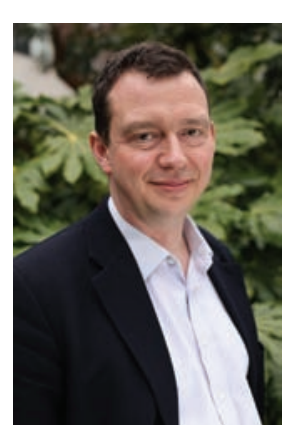

I have a particular interest in overcoming the artificial barriers between mental and physical healthcare - palliative care is a great example where this has - to a large extent - happened successfully. I am excited that BMJ Group has invested in this journal - it gives the field a wel deserved boost to have a big name in medical publishing showing interest, and if it shares the values of other BMJ journals it will not only inform, but also inspire and entertain both clinicians and researchers.

\section{Matthew Hotopf}

Professor of General Hospital Psychiatry

Institute of Psychiatry, King's College London

Consultant Liaison Psychiatrist, St Christopher's Hospice, King's College Hospital and South London and Maudsley NHS Foundation Trust 